\title{
ON APPROXIMATION OF FUNCTIONS AND THEIR DERIVATIVES BY QUASI-HERMITE INTERPOLATION
}

\author{
G. MIN \\ CECM, Department of Mathematics and Statistics \\ Simon Fraser University \\ Burnaby, B.C., Canada V5A 1S6
}

(Received April 2, 1991 and in revised form April 18, 1994)

\begin{abstract}
In this paper, we consider the simultaneous approximation of the derivatives of the functions by the corresponding derivatives of quasi-Hermite interpolation based on the zeros of (1$\left.x^{2}\right) p_{n}(x)$ (where $p_{n}(x)$ is a Legendre polynomial). The corresponding approximation degrees are given. . It is shown that this matrix of nodes is almost optimal.
\end{abstract}

KEY WORDS: IIermite interpolation, optimal nodes, derivatives, Legendre polynomials, best approximation.

1991 MATHEMATICS SUBJECT CLASSIFICATION: $41 \mathrm{~A} 05,41 \Lambda 25,41 \Lambda 28$.

\section{INTRODUCTION.}

Let

$$
-1 \leq x_{n}<\ldots<x_{1}<x_{0} \leq 1
$$

be an arbitrary nodes system on $[-1,1]$ and let $f \in C^{1}[-1,1]$. We consider the Ilermite interpolation opcrator:

$$
H_{n}(f, x):=\sum_{k=0}^{n} f\left(x_{k}\right) h_{k}(x)+\sum_{k=0}^{n} f^{\prime}\left(x_{k}\right) \sigma_{k}(x)
$$

where

$$
\begin{gathered}
h_{k}(x)=v_{k}(x) l_{k}^{2}(x), \quad \sigma_{k}(x)=\left(x-x_{k}\right) l_{k}^{2}(x), \\
l_{k}(x)=\frac{\omega(x)}{\omega^{\prime}\left(x_{k}\right)\left(x-x_{k}\right)}, \\
v_{k}(x)=1-\frac{\omega^{\prime \prime}\left(x_{k}\right)}{\omega^{\prime}\left(x_{k}\right)}\left(x-x_{k}\right), \quad \omega(x)=\Pi_{k=0}^{n}\left(x-x_{k}\right) .
\end{gathered}
$$

It satisfies the following conditions:

$$
H_{n}\left(f, x_{k}\right)=f\left(x_{k}\right), \quad k=0,1, \ldots, n
$$

and

$$
H_{n}^{\prime}\left(f, x_{k}\right)=f^{\prime}\left(x_{k}\right), \quad k=0,1, \ldots, n
$$

There have been many articles considering the problem of approximation to $f(x)$ by $H_{n}(f, x)$, Generally, we consider approximation of $f^{\prime}(x)$ by the derivative of Hermite interpolation. We know that the convergence

$$
\lim _{n \rightarrow \infty}\left\|H_{n}^{\prime}(f, x)-f^{\prime}(x)\right\|=0
$$

does not hold for all $f \in C^{1}[-1,1]$ (here $\|$.$\| is the maximum norm). Pottinger [1] investigated this$ problem when $\left\{x_{k}\right\}_{k=0}^{n}$ are the zeros of the Tchebycheff polynomial of the first kind and obtained the following result: 


$$
\left\|H_{n}^{\prime}(f, x)-f^{\prime}(x)\right\|=O(n) E_{2 n}\left(f^{\prime}\right),
$$

where $E_{n}(f)$ is the best approximation of $\int(x)$. (The factor $O(n)$ is best possible, cf. Stcinhaus [2].) In [3], Szabados and Varma introduced a norm for the higher derivatives of the operator (1.2):

$$
\left\|I_{n}^{(r)}\right\|=\sup \left\{\left\|I I_{n}^{(r)}(f, x)\right\|:\left|f^{(\imath)}\left(x_{k}\right)\right| \leq n^{2}\left(1-x_{k}^{2}\right)^{r-\imath / 2}, k=1, \ldots, n ; \imath=0,1\right\}
$$

$(r, n=1,2, \ldots)$ and they proved that for any system of nodes ([3, Theorem 1$])$

$$
\left\|H_{n}^{(r)}\right\| \geq c_{r} n^{r} \ln n, \quad(n, r=1,2, \ldots)
$$

where $c_{r}>0$ depends only on $r$. Morcover, for the matrix of nodes:

$$
\omega(x)=P_{n-2 t+1}^{(\alpha, \alpha)}(x) \Pi_{\jmath=1}^{t}\left(x^{2}-\cos ^{2} \frac{(j-1) \pi}{3 t(n-2 t+1)}\right),
$$

they obtain ([3, Theorem 3])

$$
\left\|I_{n}^{(r)}\right\|=O\left(n^{r} \ln n\right),
$$

where $t=\left[\frac{r+3}{4}\right], \alpha=2 t-\frac{r+1}{2}(r \geq 1$ integer $)$ and $P_{n-2 t+1}^{(\alpha, \alpha)}(x)$ are the ultraspherical Jacobi polynomials of degree $n-2 t$. Moreover, $\alpha$ takes only the values $-1 / 2,0,1 / 2,1$ according to $r=0,3,2,1(\bmod 4)$. (See [3, Remark, P305].) Therefore for the matrix of nodes defined by (1.5) we have

$$
\left\|H_{n}^{(r)}(f, x)-f^{(r)}(x)\right\|=O(\ln n) \omega\left(f^{(r)}, \frac{1}{n}\right) .
$$

(see [3]) At the end of paper [3], they speculated that "it would be interesting to construct a matrix which is optimal for all the derivatives up to order $r$." This is the problem of constructing matrix nodes so that the corresponding simultaneous approximation of $f(x)$ from the first derivative to the $r$-th derivative is optimal by the corresponding IIermite interpolation.

Remark: With respect to Lagrange interpolation, the complete solution of minimizing the corresponding derivatives norm to (1.4) was given by Szabados [4] (also see Vértesi [5]). The main idea is that adding nodes (near \pm 1 ) to Jacobi nodes make the similar estimates of (1.4) optimal.

In this paper, we point out that for the quasi-IIermite interpolation $R_{n}(f, x)$ based on the zeros of $\left(1-x^{2}\right) p_{n}(x)$ (where $p_{n}(x)$ is the Legendre polynomial with normalization: $p_{n}(1)=1$ ), we have

THEOREM 1. If $f \in C^{1}[-1,1]$, then

$$
\left\|R_{n}^{\prime}(f, x)-f^{\prime}(x)\right\|=O(\ln n) E_{2 n}\left(f^{\prime}\right) .
$$

THEOREM 2. If $f \in C^{r}[-1,1](r \geq 2)$, then

$$
\begin{gathered}
\left\|R_{n}^{\prime}(f, x)-f^{\prime}(x)\right\|=O(\ln n) E_{2 n}\left(f^{\prime}\right)=O\left(\frac{\ln n}{n}\right) E_{2 n-1}\left(f^{\prime \prime}\right), \\
\left\|\sqrt{1-x^{2}}\left(R_{n}^{\prime \prime}(f, x)-f^{\prime \prime}(x)\right)\right\|=O(\ln n) E_{2 n-1}\left(f^{\prime \prime}\right),
\end{gathered}
$$

and

$$
\left\|R_{n}^{(\mathfrak{\imath})}(f, x)-f^{(\mathfrak{\imath})}(x)\right\|_{[-\sigma, \sigma]}=O(\ln n) E_{2 n-\imath+1}\left(f^{(\mathfrak{\imath})}\right), \quad i=2, \ldots, r
$$

where $0<\sigma<1$.

From this we see that the zeros of $\left(1-x^{2}\right) p_{n}(x)$ are almost optimal and the corresponding simultaneous approximation is better than that of Hermite interpolation based on the zeros of Tchebysheff polynomial of the first kind.

Remark: We conjucture that the factor $\sqrt{1-x^{2}}$ in (1.10) cannot be removed on the whole interval $[-1,1]$, in which case the preceding results are optimal. 


\section{LEMMAS.}

In order to prove the Theorems, we state some properties of Legendre polynomials (sec Szegö [6]).

$$
\begin{gathered}
\left|p_{n}(x)\right| \leq 1, \\
\left(1-x^{2}\right)^{1 / 4}\left|p_{n}(x)\right| \leq(2 / \pi n)^{-1 / 2}, \quad n \geq 2 \\
\left(1-x^{2}\right)^{3 / 4}\left|p_{n}^{\prime}(x)\right| \leq(2 n)^{1 / 2}, \quad n \geq 3 \\
\sin ^{2} \theta_{k}=1-x_{k}^{2}>(k-3 / 2)^{2} n^{-2}, \quad k=1, \ldots,[n / 2] \\
\left|p_{n}^{\prime}\left(x_{k}\right)\right|>c(k-3 / 2)^{-3 / 2} n^{2}, \quad k=1, \ldots,[n / 2]
\end{gathered}
$$

We note that in (2.4) and (2.5) similar estimates are hold for $k=[n / 2], \ldots, n$. On combining (2.4) and (2.5), it follows that

$$
\left[\left(1-x_{k}^{2}\right)^{3 / 4}\left|p_{n}^{\prime}(x)\right|\right]^{2} \geq c n, \quad k=1, \ldots, n
$$

' where $c$ is an absolute positive constant independent of $f$ and $n$, whose value may vary from line to line throught our paper.

Let

$$
-1=x_{n+1}<x_{n}<\ldots<x_{1}<x_{0}=1
$$

be the zeros of $\left(1-x^{2}\right) p_{n}(x)$. Then its corresponding quasi-Hermite interpolation is the following

$$
R_{n}(f, x)=\sum_{k=0}^{n+1} f\left(x_{k}\right) r_{k}(x)+\sum_{k=1}^{n} f^{\prime}\left(x_{k}\right) \gamma_{k}(x),
$$

where

It satisfies that

$$
\begin{gathered}
r_{0}(x)=\frac{1+x}{2} p_{n}^{2}(x), \quad r_{n+1}=\frac{1-x}{2} p_{n}^{2}(x) \\
r_{k}(x)=\frac{1-x^{2}}{1-x_{k}^{2}} l_{k}^{2}(x), \quad k=1, \ldots, n \\
\gamma_{k}(x)=\left(x-x_{k}\right) r_{k}(x), \quad k=1, \ldots, n \\
l_{k}(x)=\frac{p_{n}(x)}{p_{n}^{\prime}\left(x_{k}\right)\left(x-x_{k}\right)}, \quad k=1, \ldots, n
\end{gathered}
$$

and

$$
R_{n}\left(f, x_{k}\right)=f\left(x_{k}\right), \quad k=0,1, \ldots, n+1 .
$$

LEMMA 1. We have

$$
R_{n}^{\prime}\left(f, x_{k}\right)=f^{\prime}\left(x_{k}\right), \quad k=1, \ldots, n
$$

$$
\sqrt{1-x_{k}^{2}} \leq \sqrt{1-x^{2}}+2 \frac{\left|x-x_{k}\right|}{\sqrt{1-x_{k}^{2}}}, \quad k=1, \ldots, n .
$$

PROOF. One easily sees that

$$
\begin{gathered}
\sqrt{1-x_{k}^{2}}=\sqrt{1-x^{2}}+\sqrt{1-x_{k}^{2}}-\sqrt{1-x^{2}} \\
=\sqrt{1-x^{2}}+\frac{x^{2}-x_{k}^{2}}{\sqrt{1-x_{k}^{2}}+\sqrt{1-x^{2}}} \leq \sqrt{1-x^{2}}+2 \frac{\left|x-x_{k}\right|}{\sqrt{1-x_{k}^{2}}} .
\end{gathered}
$$

This proves Lemma 1. 
LEMMA 2. We have

$$
\begin{gathered}
\text { (i) } I_{1}:=\sum_{k=1}^{n} \frac{\left|x-x_{k}\right|}{1-x_{k}^{2}} l_{k}^{2}(x)=O(\ln n) \\
\text { (ii) } I_{2}:=\sum_{k=1}^{n}\left|x-x_{k}\right| \frac{1-x^{2}}{1-x_{k}^{2}}\left|l_{k}(x) l_{k}^{\prime}(x)\right|=O(\ln n)
\end{gathered}
$$

PROOF. From Lemma 1 we have

$$
I_{1} \leq \sum_{k=1}^{n} \frac{\sqrt{1-x^{2}}\left|x-x_{k}\right|}{\left(1-x_{k}^{2}\right)^{3 / 2}} l_{k}^{2}(x)+2 \sum_{k=1}^{n} \frac{\left|x-x_{k}\right|^{2}}{\left(1-x_{k}^{2}\right)^{2}} l_{k}^{2}(x):=A_{1}(x)+A_{2}(x)
$$

Throughout this paper we assume $x$, to be the zero of $p_{n}(x)$ which is the nearest to $x$ and $i=|k-j|$. By using (5.8) in Prasad and Varma[7] we have

$$
\sqrt{1-x^{2}} \frac{\left|x-x_{\jmath}\right|}{1-x_{\jmath}^{2}} l_{\jmath}^{2}(x) \leq \frac{c}{n} .
$$

Notice that, with $x=\cos \theta \quad(0 \leq \theta \leq \pi)$

$$
\sin \theta \leq \sin \theta+\sin \theta_{k} \leq 2 \sin \frac{\theta+\theta_{k}}{2}
$$

so we have

$$
\begin{aligned}
& A_{1}(x)=\frac{1}{\sqrt{1-x_{\jmath}^{2}}} \frac{\sqrt{1-x^{2}}\left|x-x_{\jmath}\right|}{1-x_{\jmath}^{2}} l_{\jmath}^{2}(x)+\sum_{k \neq j} \frac{\sqrt{1-x^{2}}\left|x-x_{k}\right|}{\left(1-x_{k}^{2}\right)^{3 / 2}} l_{k}^{2}(x) \\
& \leq \frac{c}{n} \frac{1}{\sin \theta_{\jmath}}+\sum_{k \neq j} \frac{\sqrt{1-x^{2}} p_{n}^{2}(x)}{\left[\left(1-x_{k}^{2}\right)^{3 / 4}\left|p_{n}^{\prime}\left(x_{k}\right)\right|\right]^{2}\left|x-x_{k}\right|} \\
& =O(1)\left[1+p_{n}^{2}(x) \sum_{k \neq j} \frac{1}{\sin \left|\frac{\theta-\theta_{k}}{2}\right|}\right]=O(1)\left[1+\frac{p_{n}^{2}(x)}{n} \sum_{k \neq j} \frac{n}{i}\right]=O(\ln n) .
\end{aligned}
$$

Similarly,

$$
A_{2}(x)=\sum_{k=1}^{n} \frac{p_{n}^{2}(x)}{\left[\left(1-x_{k}^{2}\right)^{3 / 4}\left|p_{n}^{\prime}\left(x_{k}\right)\right|\right]^{2} \sqrt{1-x_{k}^{2}}}=O(1) \frac{p_{n}^{2}(x)}{n} \sum_{k=1}^{n} \frac{1}{\sqrt{1-x_{k}^{2}}}=O(\ln n)
$$

so we obtain (2.8).

Notice that

and we have

$$
l_{k}^{\prime}(x)=\frac{p_{n}^{\prime}(x)\left(x-x_{k}\right)-p_{n}(x)}{\left(x-x_{k}\right)^{2} p_{n}^{\prime}\left(x_{k}\right)}
$$

$$
I_{2} \leq \sum_{k=1}^{n}\left|x-x_{k}\right| \frac{\left(1-x^{2}\right)\left|x-x_{k}\right|\left|p_{n}^{\prime}(x)\right|}{\left(1-x_{k}^{2}\right)\left(x-x_{k}\right)^{2}\left|p_{n}^{\prime}\left(x_{k}\right)\right|}\left|l_{k}(x)\right|+\sum_{k=1}^{n} r_{k}(x):=B_{1}(x)+B_{2}(x)
$$

One notes Prasad and Varma [7]

$$
\frac{\left(1-x^{2}\right)^{1 / 4}}{\left(1-x_{k}^{2}\right)^{1 / 4}}\left|l_{k}(x)\right| \leq c
$$

so we have

$$
\begin{gathered}
B_{1}(x)=\sum_{k=1}^{n} \frac{\left(1-x^{2}\right)^{3 / 4}\left|p_{n}^{\prime}(x)\right|}{\left(1-x_{k}^{2}\right)^{3 / 4}\left|p_{n}^{\prime}\left(x_{k}\right)\right|} \frac{\left(1-x^{2}\right)^{1 / 4}}{\left(1-x_{k}^{2}\right)^{1 / 4}}\left|l_{k}(x)\right| \\
=O(1) \frac{\left(1-x^{2}\right)^{3 / 4}\left|p_{n}^{\prime}(x)\right|}{\left(1-x_{\jmath}^{2}\right)^{3 / 4}\left|p_{n}^{\prime}\left(x_{\jmath}\right)\right|}+\sum_{k \neq j} \frac{\left(1-x^{2}\right)\left|p_{n}(x) p_{n}^{\prime}(x)\right| \sqrt{1-x_{k}^{2}}}{\left[\left(1-x_{k}^{2}\right)^{3 / 4}\left|p_{n}^{\prime}\left(x_{k}\right)\right|\right]^{2}\left|x-x_{k}\right|} \\
=O(1)\left[1+\frac{\left(1-x^{2}\right)\left|p_{n}(x) p_{n}^{\prime}(x)\right|}{n} \sum_{k \neq j} \frac{\sin \theta_{k}}{\left|x-x_{k}\right|}\right] \\
=O(1)\left[1+\ln n\left(1-x^{2}\right)\left|p_{n}(x) p_{n}^{\prime}(x)\right|\right]=O(\ln n) .
\end{gathered}
$$


Obviously,

$$
B_{2}(x) \leq \sum_{k=0}^{n+1} r_{k}(x) \equiv 1
$$

Therefore we obtain (2.9).

LEMMA 3. We have

$$
I_{3}:=\sum_{k=0}^{n+1}\left(1-x_{k}^{2}\right)\left|r_{k}(x)\right|=O(\ln n)\left(1-x^{2}\right),
$$

and

$$
I_{4}:=\sum_{k=1}^{n} \sqrt{1-x_{k}^{2}}\left|\gamma_{k}(x)\right|=O\left(\frac{\ln n}{n}\right) \sqrt{1-x^{2}}
$$

Proof. Since

$$
I_{3}=\left(1-x^{2}\right) \sum_{k=1}^{n} l_{k}^{2}(x)
$$

from Nevai and Vértesi [8] we have

$$
\sum_{k=1}^{n} l_{k}^{2}(x)=O(1)\left(1+\frac{J_{n}^{2}(x)}{n}+\frac{\ln n}{n} J_{n}^{2}(x)\right),
$$

where $J_{n}(x)$ is the orthonormal Legendre polynomials:

$$
\int_{-1}^{1} J_{n}(x) J_{m}(x) d x=\delta_{n m}
$$

and notice that Natanson [9] gives

$$
\left\|J_{n}(x)\right\|=O(1) n^{1 / 2}
$$

It follows that

$$
\sum_{k=1}^{n} l_{k}^{2}(x)=O(\ln n)
$$

this implies (2.12). Also, we have

$$
\begin{gathered}
I_{4}=\sum_{k=1}^{n} \frac{\left(1-x^{2}\right)\left|x-x_{k}\right|}{\sqrt{1-x_{k}^{2}}} l_{k}^{2}(x) \\
=\left(1-x^{2}\right) \frac{\left(1-x_{\jmath}^{2}\right)^{1 / 4}\left|p_{n}(x)\right|}{\left(1-x_{\jmath}^{2}\right)^{3 / 4}\left|p_{n}^{\prime}\left(x_{\jmath}\right)\right|}\left|l_{\jmath}(x)\right|+\sum_{k \neq j} \frac{\left(1-x^{2}\right) p_{n}^{2}(x)}{\left[\left(1-x_{k}^{2}\right)^{3 / 4}\left|p_{n}^{\prime}\left(x_{k}\right)\right|\right]^{2}} \frac{1-x_{k}^{2}}{\left|x-x_{k}\right|} .
\end{gathered}
$$

Recall that (Erdös [10]) for $-1 \leq x \leq 1$,

$$
\left|l_{k}(x)\right| \leq 1, \quad k=1, \ldots, n
$$

therefore, similar to the estimates of $I_{1}$ and $I_{2}$, we have

$$
I_{4}=O(1) \frac{1-x^{2}}{n}+\frac{\left(1-x^{2}\right) p_{n}^{2}(x)}{n} \sum_{k \neq j} \frac{1}{\sin \left|\frac{\theta-\theta_{k}}{2}\right|}=O\left(\frac{\ln n}{n}\right) \sqrt{1-x^{2}} .
$$

This proves Lemma 3.

Remark: If we need not want to obtain the factor $\left(1-x^{2}\right)$, we can obtain a better estimate of $I_{3}$.

LEMMA 4. Let $f \in C^{r}[-1,1]$, then there exist polynomials $q_{n}(x)$ of degree $n \geq 4 r+5$ such that $(j=0,1, \ldots, r)$

$$
\left|f^{(\jmath)}(x)-q_{n}^{(\jmath)}(x)\right|=O(1)\left(\frac{\sqrt{1-x^{2}}}{n}\right)^{r-\jmath} E_{n-r}\left(f^{(r)}\right)
$$


PROOF. Irom Gopengauy's Theorem [11] we know that there exist polynomials $l_{n}(x)$ of degree $n \geq$ $4 r+5$ such that

$$
\left|f^{(\jmath)}(x)-t_{n}^{(\jmath)}(x)\right| \leq c\left(\frac{\sqrt{1-x^{2}}}{n}\right)^{r-\jmath} \omega\left(f^{(r)}, \frac{\sqrt{1-x^{2}}}{n}\right)
$$

Let $s_{n}(x)$ be the polynomial of degree $n>r$ such that

$$
\left\|f^{(r)}(x)-s_{n}^{(r)}(x)\right\| \leq E_{n-r}\left(f^{(r)}\right),
$$

then we have

$$
\begin{gathered}
\left|f^{(\jmath)}(x)-q_{n}^{(\jmath)}(x)\right|:=\left|f^{((\jmath)}(x)-\left(s_{n}^{(\jmath)}(x)+t_{n}^{(\jmath)}(x)\right)\right| \\
\leq c\left(\frac{\sqrt{1-x^{2}}}{n}\right)^{r-\jmath} \omega\left(\left(\int-s_{n}\right)^{(r)}, \frac{1}{n}\right)=O(1)\left(\frac{\sqrt{1-x^{2}}}{n}\right)^{r-\jmath}\left\|f^{(r)}-s_{n}^{(r)}\right\| \\
=O(1)\left(\frac{\sqrt{1-x^{2}}}{n}\right)^{r-\jmath} E_{n-r}\left(f^{(r)}\right) .
\end{gathered}
$$

This proves Lemma 4 .

LEMMA 5. Let $s_{\jmath}(x)$ be a polynomial of degrec $\leq n$, and suppose that the inequality

$$
\sum_{j=1}^{m}\left|s_{\jmath}(x)\right|=O(1), \quad-1 \leq x \leq 1 .
$$

holds. Then

$$
\left(1-x^{2}\right)^{2 / 2} \sum_{\jmath=1}^{m}\left|s_{\jmath}^{(2)}(x)\right|=O(1) n^{2}
$$

where $m \geq 1$ and $1 \leq i \leq n$.

PROOF. Although Ramm [12, Lcmma 1, p285] only proved the case of $i=1,(26)$ can be obtained by using a completely similar method.

\section{PROOFS OF THEOREMS.}

PROOF OF THEOREM 1 . Notice that

$$
\begin{aligned}
R_{n}(f, x) & -f(x)=\sum_{k=0}^{n+1}\left(f\left(x_{k}\right)-f(x)\right) r_{k}(x)+\sum_{k=1}^{n} f^{\prime}\left(x_{k}\right) \gamma_{k}(x) \\
& =\sum_{k=0}^{n+1} \int_{x}^{x_{k}} f^{\prime}(t) d t r_{k}(x)+\sum_{k=1}^{n} f^{\prime}\left(x_{k}\right) \gamma_{k}(x) .
\end{aligned}
$$

This implies

$$
\left\|R_{n}^{\prime}\right\| \leq\left(\sum_{k=0}^{n+1}\left|x-x_{k}\right| r_{k}^{\prime}(x)\left|+\sum_{k=1}^{n}\right| \gamma_{k}^{\prime}(x) \mid\right)\left\|f^{\prime}\right\|
$$

One easily sees that

$$
(1-x)\left|r_{0}^{\prime}(x)\right| \leq(1-x)\left[\frac{p_{n}^{2}(x)}{2}+(1+x)\left|p_{n}(x) p_{n}^{\prime}(x)\right|\right]=O(1) .
$$

Similarly we have

$$
(1+x)\left|r_{n+1}^{\prime}(x)\right|=O(1)
$$

Notice that

$$
r_{k}^{\prime}(x)=-\frac{2 x}{1-x_{k}^{2}} l_{k}^{2}(x)+\frac{2\left(1-x^{2}\right)}{1-x_{k}^{2}} l_{k}(x) l_{k}^{\prime}(x)
$$

and

$$
\gamma_{k}^{\prime}(x)=r_{k}(x)+\left(x-x_{k}\right) r_{k}^{\prime}(x)
$$

From Lemma 2 we have 


$$
\sum_{k=0}^{n+1}\left|x-x_{k}\right|\left|r_{k}^{\prime}(x)\right|=O(\ln n)
$$

and also we have

$$
\sum_{k=1}^{n}\left|\gamma_{k}^{\prime}(x)\right|=O(\ln n)
$$

It now follows that

$$
\left\|R_{n}^{\prime}\right\|=O(\ln n)\left\|f^{\prime}\right\| .
$$

Combining Lemma 4, (3.2) and (3.3), we obtain Theorem 1.

PROOF OF THEOREM 2. Theorem 1 implies (9). Ilere we only prove the case $i=2$. The other cases are completely similar. By using Lemma 5 (or sec Borwcin and Erdelyi [13]) and from Lemma 3 we obtain the following

$$
\sum_{1}^{n+1}\left(1-x_{k}^{2}\right)\left|r_{k}^{\prime \prime}(x)\right|=O\left(n^{2} \ln n\right)
$$

and

$$
\sqrt{1-x^{2}} \sum_{k=1}^{n} \sqrt{1-x_{k}^{2}}\left|\gamma_{k}^{\prime \prime}(x)\right|=O(n \ln n)
$$

Notice that

$$
R_{n}^{\prime \prime}(f, x)-f^{\prime \prime}(x)=R_{n}^{\prime \prime}\left(f-q_{2 n+1}, x\right)+q_{2 n+1}^{\prime \prime}(x)-f^{\prime \prime}(x)
$$

and

$$
R_{n}^{\prime \prime}\left(f-q_{2 n+1}, x\right)=\sum_{k=0}^{n+1}\left(f\left(x_{k}\right)-q_{2 n+1}\left(x_{k}\right)\right) r_{k}^{\prime \prime}(x)+\sum_{k=1}^{n}\left(f^{\prime}\left(x_{k}\right)-q_{2 n+1}^{\prime}\left(x_{k}\right)\right) \gamma_{k}^{\prime \prime}(x) .
$$

Combining Lemma $4,(3.5)$ and (3.6), we obtain (1.10).

ACKNOWLEDGEMENT: The author thanks Professor P. Borwein and referees for their valuable suggestions and comments.

\section{References}

[1] Pottinger, P., On the approximation of functions and their derivatives by IIermit e interpolation , J. Approx. Th., 23(1978), 267-273.

[2] Steinhaus, B., On the $C^{1}$-norm of the Hermite interpolation, J. Approx. T h., $\underline{50}(1987), 160-166$.

[3] Szabados, J., and Varma, A. K., On the derivatives of Hermite-Fejér interpolat ing polynomials, Acta Math. Hung., 55(1990), 301-309.

[4] Szabados, J., On the convergence of the derivatives of projection operators, Analysis, $\underline{7}(1987)$, 349-357.

[5] Vértesi, P., Derivatives of projection operators, Analysis, $\underline{9}(1989)$, 145-156.

[6] Szegö, G., Orthogonal polynomials, American Math. Soc. Coll. Publ., New York, 1979.

[7] Prasad, J. and Varma, A. K., A study of some interpolatory processes based on th e roots of Legendre polynomials, J. Approx. Th., $\underline{31}(1981), 244-252$.

[8] Nevai, P., and Vértesi, P., Mean convergence of Iỉermite-Fejér interpolation, J. Math. Anal. Appl., $\underline{105}(1985), 26-58$. 
[9] Natanson, I. P., Construclive funclion thcory, Vol. II, Ungar, New York, 1965 .

[10] Erdös, P., On the maximum of the fundamental functions of the ultraspherical polynomials, $A n n$. of Malh., 15(1944), 335-339.

[11] Gopengauz, I. E., On a theorem of $\Lambda$. F. Timan on approximation of functions by polynomials on a finite interval, Mat. Zamelki, 1(1967), 163-172.

[12] Ramm, A. G., On simultancous approximation of a function and its derivative by interpolation polynomials, Bull. London. Math. Soc., 2(1977), 283-288.

[13] Borwcin, P., and Erdeli, T., Polynomials and polynomials incqualitics, Springer-Verlag, (to appear) 


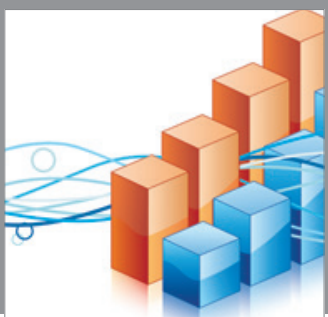

Advances in

Operations Research

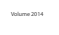

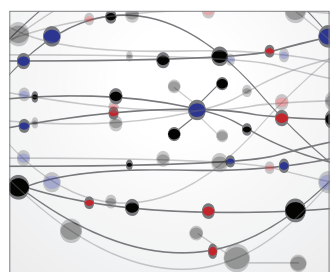

\section{The Scientific} World Journal
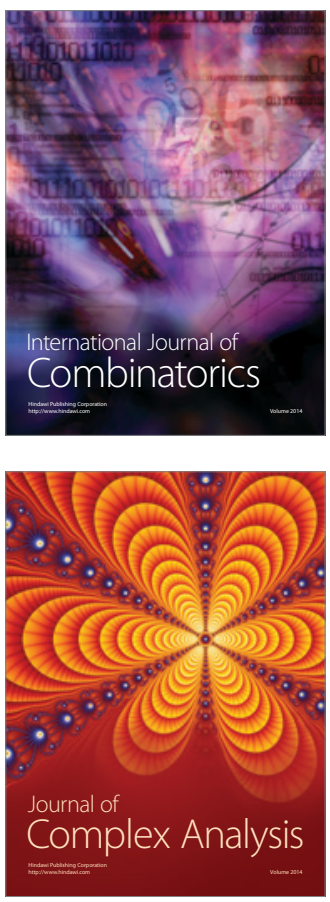

International Journal of

Mathematics and

Mathematical

Sciences
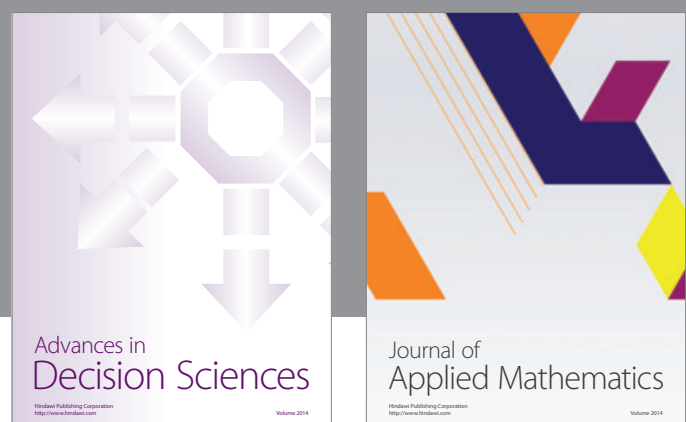

Journal of

Applied Mathematics
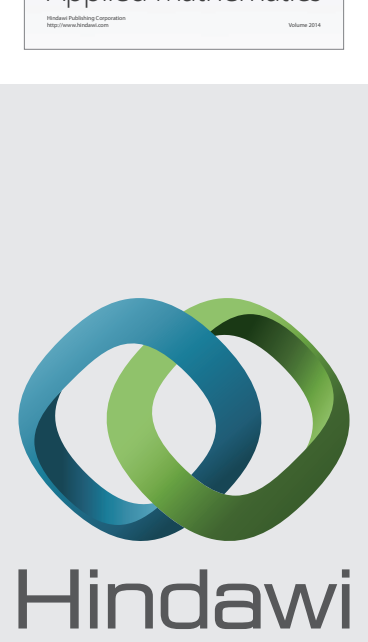

Submit your manuscripts at http://www.hindawi.com
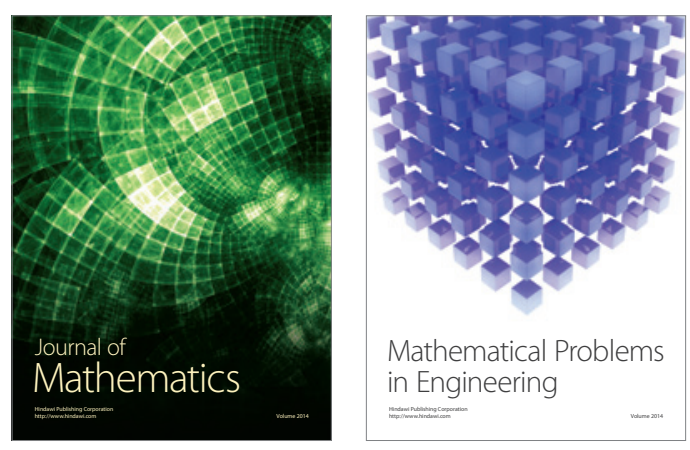

Mathematical Problems in Engineering
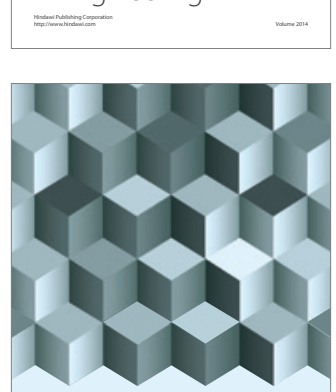

Journal of

Function Spaces
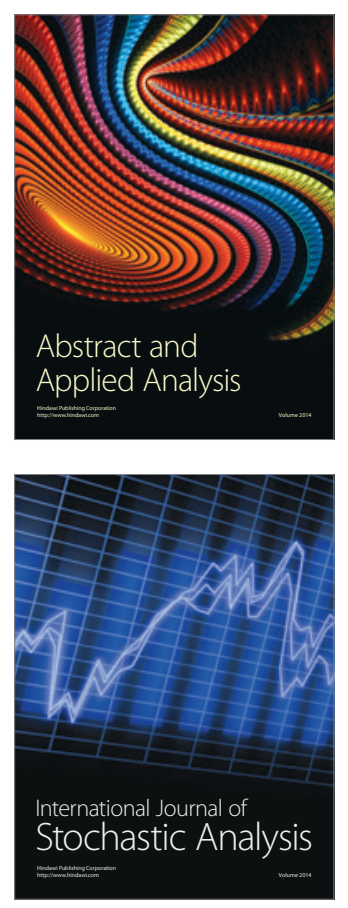

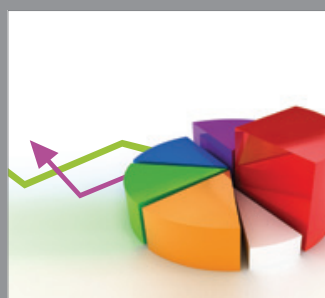

ournal of

Probability and Statistics

Promensencen
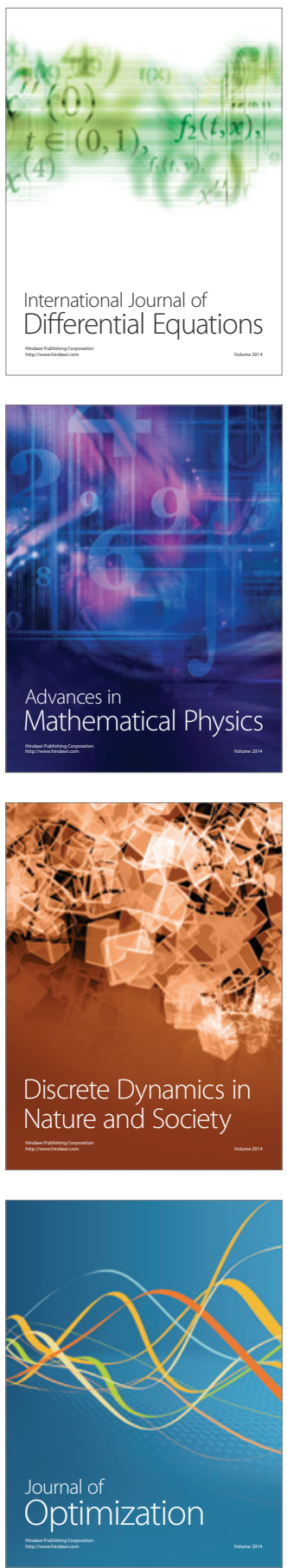